\title{
Evidence for a defect in fatty acid uptake by adipose tissue of patients with hypertriglyceridaemia ${ }^{1}$
}

\author{
LARS A. CARLSON, GÖRAN WALldiUS, AND ANDERS G. OLSSON \\ From the Department of Geriatrics, University of Uppsala, and King Gustaf Vth Research Institute, \\ Stockholm, Sweden
}

Professor Robinson has described the importance of lipoprotein lipase (clearing factor lipase) for the initial phase in the clearance of plasma triglycerides, whether exogenous and present as chylomicrons or endogenous and present as very-low-density lipoprotein (VLDL). It acts by splitting off the fatty acids which are then taken up by the cells. Our interest in hypertriglyceridaemia was extended to the later phases of the clearance process as a result of an unusual case described below.

Case A.A. was a man aged 50 with massive hypertriglyceridaemia, with plasma levels sometimes exceeding $150 \mathrm{mmol} / \mathrm{l}^{2}$ (approximately 12500 $\mathrm{mg} / 100 \mathrm{ml}$ ) compared with the normal of about 1-2 $\mathrm{mmol} / \mathrm{l}$. Initially, fasting plasma looked like cream and most of the triglyceride was present in the chylomicrons, ie, in particles with $\mathrm{Sf}>400$, but there was also elevation of the VLDL with $\mathrm{Sf}<400$ (table I). The hyperlipidaemia is thus best classified

\begin{tabular}{|c|c|c|c|c|c|c|}
\hline & \multicolumn{5}{|c|}{ Fraction $^{1}$} & \multirow{2}{*}{$\begin{array}{l}\text { Total } \\
\text { VLDL }\end{array}$} \\
\hline & $A$ & $B$ & $C$ & $D$ & $E$ & \\
\hline$S_{\ell}$ range & $>5000$ & $\begin{array}{l}400 \\
\text { to } \\
5000\end{array}$ & $\begin{array}{l}100 \\
\text { to } \\
400\end{array}$ & $\begin{array}{l}50 \\
\text { to } \\
100\end{array}$ & $\begin{array}{l}20 \\
\text { to } \\
10\end{array}$ & $>20$ \\
\hline $\begin{array}{l}\text { Triglyceride con- } \\
\text { centration }(\mathrm{mmol} / \mathrm{l})\end{array}$ & $42 \cdot 8$ & $12 \cdot 1$ & $15 \cdot 5$ & $0 \cdot 8$ & $0 \cdot 2$ & $74 \cdot 2$ \\
\hline
\end{tabular}

Table I Distribution of fasting plasma triglycerides of case A.A. in different fractions of the very low density lipoproteins (VLDL) separated at density 1006 according to Gustafson, Alaupovic, and Furman (1965)

${ }^{1}$ Fractions $\mathrm{A}$ and $\mathrm{B}$ comprise the chylomicrons.

as type V(World Health Organization Memorandum, 1970).

An example of the large fluctuation in plasma triglyceride concentration that occurred with variations in the diet is given in figure 1 . The patient was extremely fat-sensitive and on a fat-rich diet plasma

'Supported by grants from the Swedish Medical Research Council (19x-204)

${ }^{1} 1 \mathrm{mmol} / \mathrm{l}=88.5 \mathrm{mg} / 100 \mathrm{ml}$ for triolein triglycerides increased almost to $200 \mathrm{mmol} / 1$ (or $\stackrel{s}{\vec{c}}$ about $17500 \mathrm{mg} / 100 \mathrm{ml}$ ). However, a fat-poor diet, ć and even starvation, did not bring the level below $\overrightarrow{-}$ $50 \mathrm{mmol} / 1$, and over a period of several years the $\stackrel{\overrightarrow{ }}{\circ}$ level has usually fluctuated between about 50 and 을 $150 \mathrm{mmol} / \mathrm{l}$. Although alimentary lipaemia was exaggerated it was rapidly cleared by intravenous heparin, the free fatty acid concentration in the plasma increasing at the same time. Moreover, the plasma lipolytic activity 45 minutes after intravenous heparin (Boberg and Carlson, 1964) has been assayed $\varphi$ on several occasions, with plasma triglyceride con- $\omega$ centrations varying from 6 to $90 \mathrm{mmol} / \mathrm{l}$ depending on treatment (see below), and the results have always fallen in the range 73-124 mmol glycerol/minute $/ 1 \bar{\partial}$ compared with our normal range of 60 to $140 \%$ (Boberg, 1972). These findings suggest a defect in $\stackrel{\odot}{\AA}$ removal of triglycerides in the presence of normal $\vec{\overrightarrow{ }}$ postheparin lipoprotein lipase activity.

A point of great interest is the therapeutic response to treatment with nicotinic acid (fig 2). Not only dido the plasma triglyceride concentration fall completely, though slowly, to normal during treatment, but ito remained normal for many weeks after stopping $\overline{3}$ treatment, after which it increased slowly. A similar, though somewhat slower, response was also obtainedo with nicotinamide, which usually has no effect on hyperlipidaemia, and once again the level of tri-옥 glyceride remained almost normal for several weeks $>$ when treatment was stopped. The plasma cholesterol behaved in the same way as the triglycerides, as N would be expected from the reduction in lipoprotein? molecules (chylomicrons and VLDL) which contain 0 not only triglycerides but also cholesterol. A com- $-\omega$ plete plasma lipoprotein analysis of this case before and after treatment is given elsewhere (Carlson Fröberg, and Öro, 1972).

These observations turned our attention to $\stackrel{?}{?}$ possible explanations for a triglyceride clearance defect other than low lipoprotein lipase activity? including the possibility of a defect within the cell $\stackrel{\mathbb{\Omega}}{\Omega}$ The transport of fatty acids into the cell after $\stackrel{2}{\Omega}$ hydrolysis of plasma triglycerides by lipoproteino 


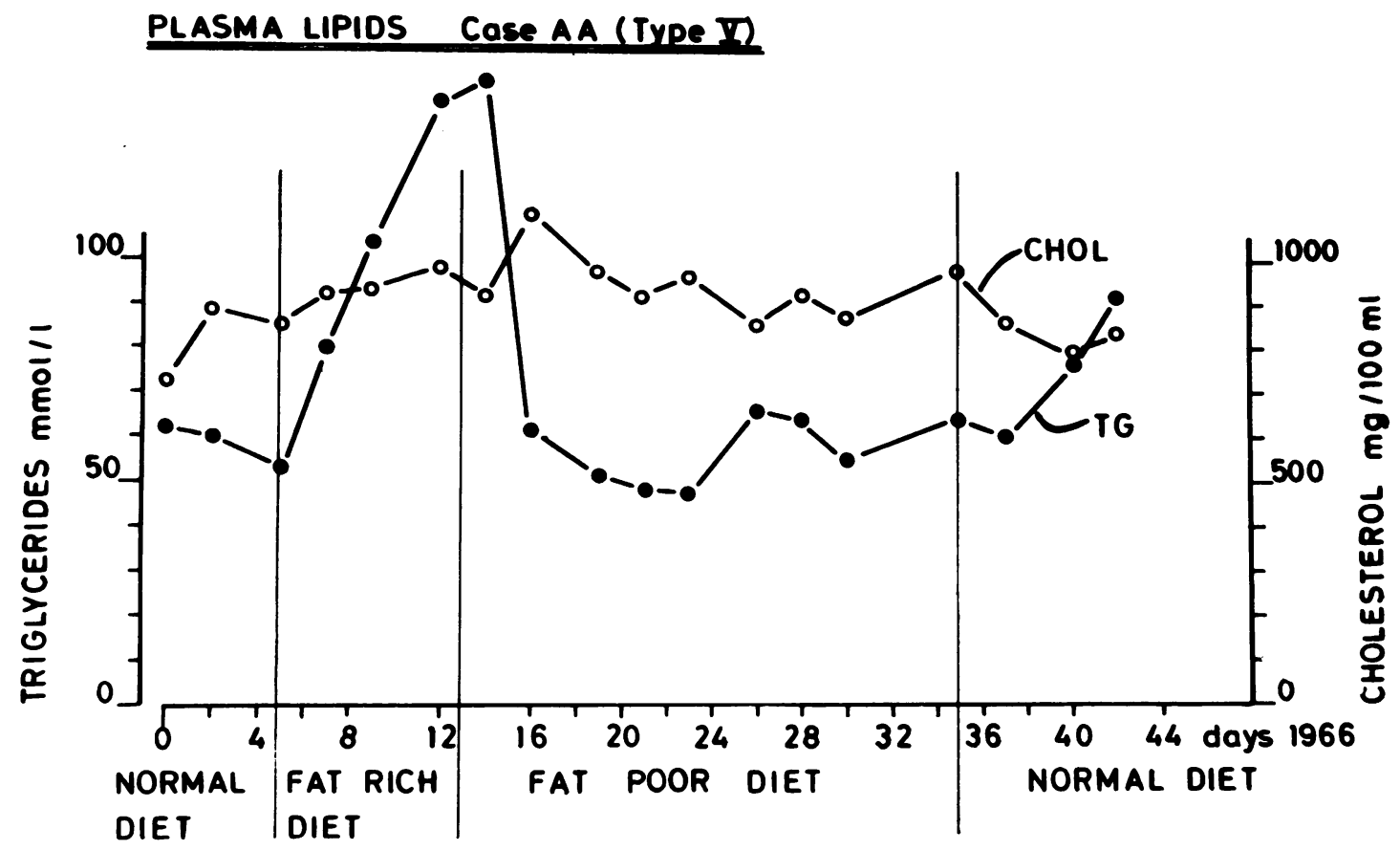

Fig 1 Variation in fasting plasma lipids with diet in case A.A.

\section{PLASMA LIPIDS Case AA (Type I)}

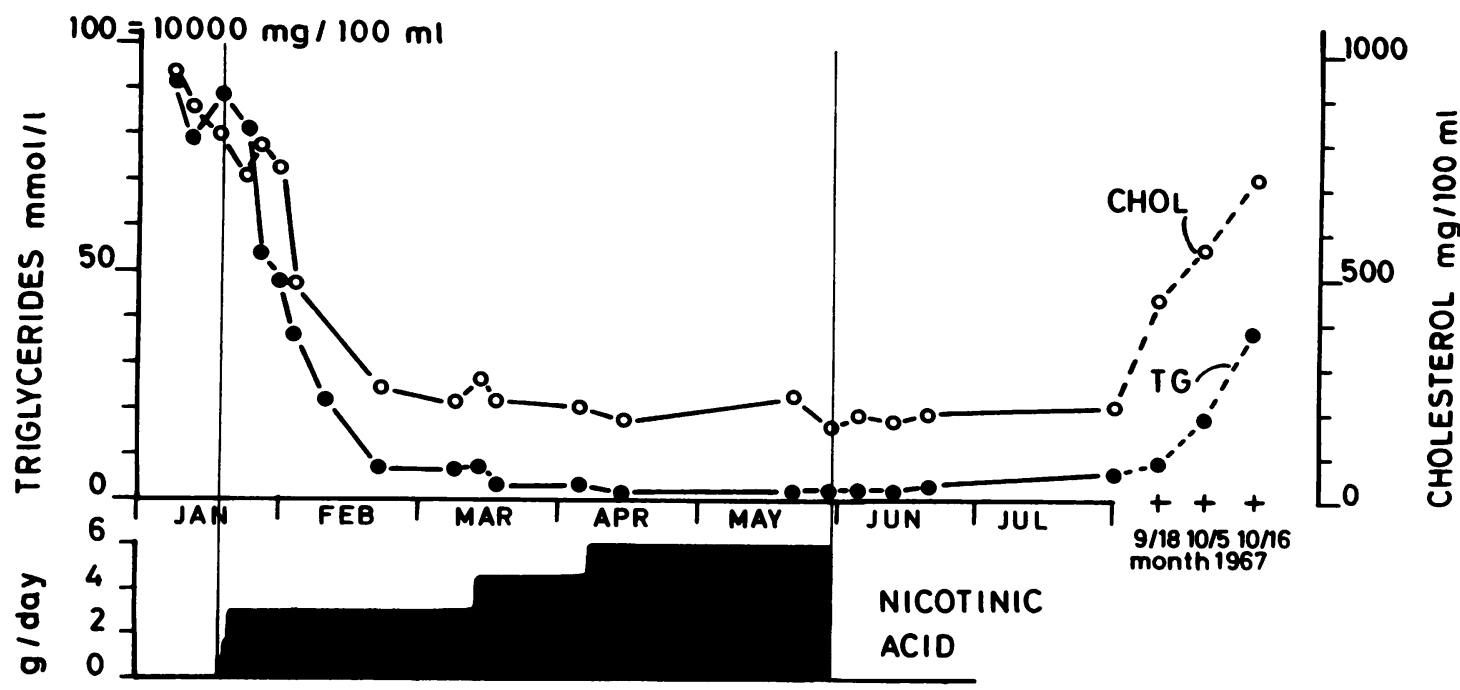

Fig 2 Effect of nicotinic acid on fasting plasma lipids in case A.A. 
lipase entails a number of reactions, involving not only the fatty acids but also glucose metabolism because $\alpha$-glycerophosphate, which is needed for the esterification of the fatty acids, is mainly formed from glucose. Figure 3 summarizes these reactions which culminate in the esterification of the fatty acids with $\alpha$-glycerophosphate and their deposition as triglycerides. It is interesting to note, in view of the patient's response to both nicotinic acid and nicotinamide, that the conversion of dihydroxyacetone phosphate (DHAP) to $\alpha$-glycerophosphate ( $\alpha$-GP) is NAD dependent. Both nicotinic acid and its amide may be precursors of NAD.

In order to explore the possibility that a defect in the processes involved in the uptake and esterification of fatty acids existed in our patient the uptake of fatty acids by adipose tissue was studied in vitro; $200 \mathrm{mg}$ of adipose tissue was incubated for two hours in a medium containing ${ }^{3} \mathrm{H}$-labelled fatty acids and ${ }^{14} \mathrm{C}$-labelled glucose (macro-method). After incubation the tissues were homogenized, the lipids separated, the glycerides hydrolysed, and the radioactivities determined in the fatty acid and glycerol moieties (Carlson, Eriksson, and Walldius, 1973). The findings in the patient both before and after treatment with nicotinic acid are given in table II together with values obtained similarly in control subjects. Before treatment the rate of incorporatio $\overrightarrow{\overrightarrow{F_{3}}}$ of both fatty acid and glucose into adipose tissuete glycerides was clearly below the lowest level observef in all control subjects. Both increased, three an six-fold respectively, to within the normal range afteo the plasma triglycerides had been restored to nea

\begin{tabular}{|c|c|c|c|c|}
\hline & \multicolumn{2}{|l|}{ Controls $^{1}$} & \multicolumn{2}{|l|}{ Case $A . A .^{2}$} \\
\hline & Normolipidaemic & Hyperlipidaemic & $\begin{array}{l}\text { Before } \\
\text { Treatment }\end{array}$ & $\begin{array}{l}\text { After } \\
\text { Treatme }\end{array}$ \\
\hline $\begin{array}{l}{ }^{3} \mathrm{H} \text {-fatty acid } \\
\mathrm{nmol} / \mathrm{g} / \mathrm{h}\end{array}$ & $\begin{array}{c}130 \div 30 \\
(70-290)\end{array}$ & $\begin{array}{l}140 \div 30 \\
(50-240)\end{array}$ & 30 & 80 \\
\hline $\begin{array}{l}{ }^{14} \mathrm{C} \text {-glucose } \\
\mathrm{nmol} / \mathrm{g} / \mathrm{h}\end{array}$ & $\begin{array}{c}310 \pm 70 \\
(180-650)\end{array}$ & $\begin{array}{c}170 \div 20 \\
(100-240)\end{array}$ & 50 & 290 \\
\hline
\end{tabular}

Table II Fatty acid and glucose incorporation into adipose tissue in vitro obtained with macromethod (mean $\pm S E M$, range)

${ }^{1}$ Tissue from controls was obtained during surgery for gallbladde disease, and from case A.A. after local anaesthesia. Controls af classified as normo- or hyperlipidaemic on the basis of blood analys performed some weeks after operation; hyperlipidaemia was on minor, and in no case was the plasma opalescent.

${ }^{2}$ Before and after treatment refers to treatment with nicotinic aci@ this was continued for two months and reduced the plasma triglycerides to near normal.

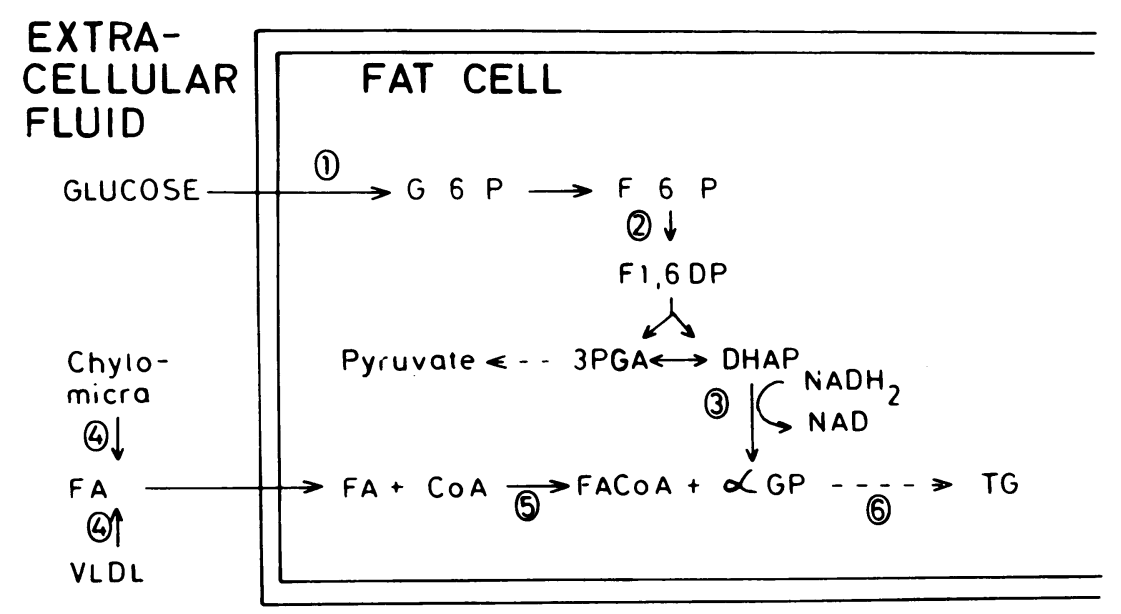

Fig 3 Schematic representation of the uptake of triglyceride fatty acids by the adipocyt

(1) Membrane transport, hexokinases

(2) Phosphofruclokinase

(3) Glycerophosphate dehydrogenase. $\mathrm{NADH}_{2} / \mathrm{NAD}$

(4) Lipoprotein lipase

(5) FA activation

(6) Triglyceride synthesis 
normal by treatment with nicotinic acid.

To summarize, in this patient there is a defect in the clearance of triglycerides from plasma. There appears to be no deficiency in lipoprotein lipase activity, but when the patient is hyperlipidaemic there is a defective uptake of fatty acids by adipose tissue; this reverts to within the normal range when plasma lipids return to near normal after treatment with nicotinic acid.

These observations led us to study the fatty acid incorporation into adipose tissue (FIAT) in vitro in other cases of hyperlipidaemia. A microtechnique was developed requiring only $20 \mathrm{mg}$ of adipose tissue obtained by fine needle biopsy, its precision being $\pm 10 \%$. The tissue is obtained by a single needle biopsy so that repeated biopsies can be taken. The results in a group of patients with hyperlipidaemia are given in figure 4 . The FIAT values in

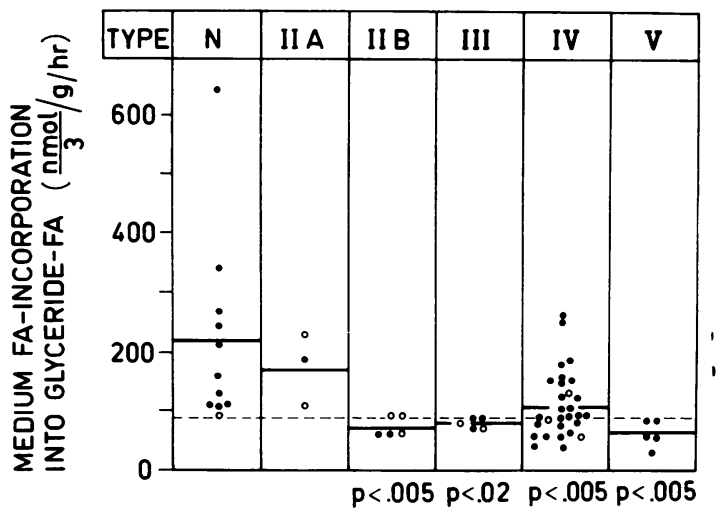

Fig 4 Fatty acid incorporation into adipose tissue $(F I A T)$ in patients with different types of hyperlipidaemia. Each symbol represents one patient. Thick bars indicate the mean, the hatched line the lowest normal value. Micromethod. P values indicate significance of difference from normals $(N)$. Men; $\bigcirc$ women.

normal subjects vary over a wide range. Five subjects with type $V$ hyperlipidaemia all had subnormal FIAT levels. In type II B, type III, and type IV all characterized by hypertriglyceridaemia, wealso found subnormal values for FIAT, while in the normotriglyceridaemic type IIA the values, as expected, were in the normal range. Figure 5 shows a similar picture for glucose incorporation into adipose tissue (GLIAT) but the differences are less pronounced.

It should be noted that several of the patients with type IV hyperlipoproteinaemia had FIAT as well as GLIAT values within the normal range. This suggests that defects other than low FIAT may be causing the

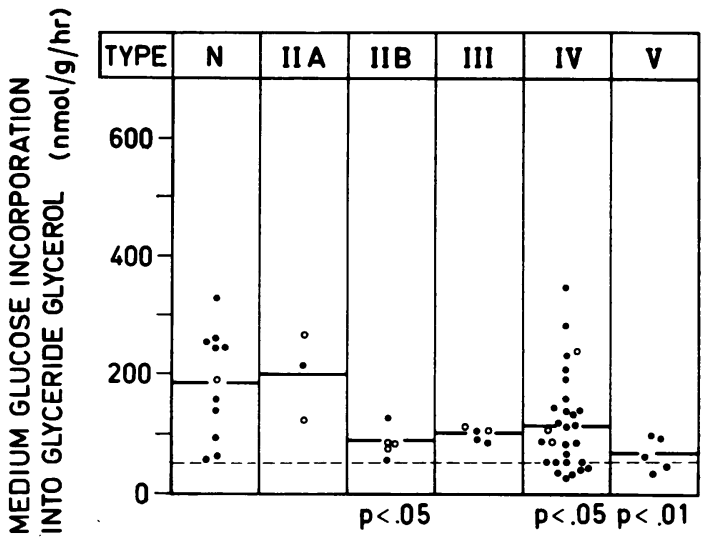

Fig 5 Incorporation of glucose into adipose tissue $(G L I A T)$ in patients with different types of hyperlipidaemia. Symbols as for figure 4.

hypertriglyceridaemia such as low lipoprotein lipase activity and increased triglyceride secretion from the liver. Another interesting feature of the adipose tissue of hyperlipidaemic subjects is that there is evidence of increased fat-mobilizing lipolysis as shown by measurement of glycerol release (fig 6). This is particularly the case in type IV.

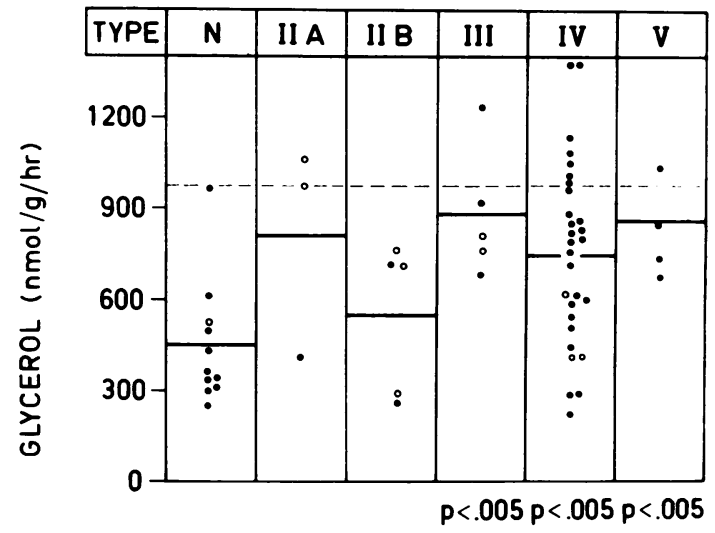

Fig 6 Lipolysis as measured by glycerol release in patients with different types of hyperlipidaemia.

Symbols as for fig 4, except that the hatched line indicates the highest normal value.

These studies have indicated a new metabolic defect-low FIAT - in adipose tissue of patients with different types of hypertriglyceridaemia. A low FIAT may possibly cause hypertriglyceridaemia by slowing down the clearance of plasma triglycerides as a result of impaired fatty acid uptake in adipose tissue. 
This new aspect on hypertriglyceridaemia may also explain a previously puzzling difference between type I and type $\mathrm{V}$ hyperlipoproteinaemia. In spite of the fact that both chylomicrons and VLDL are believed to be cleared from plasma by the same mechanisms (fig 3) it is only the former lipoprotein family which is elevated in type I while both are raised in type $V$. The finding of low FIAT in type $V$ now appears to afford an explanation for these differences. In type $I$ the defective clearing of chylomicrons is due to lack of or low lipoprotein lipase activity (fig 3), and hence no fatty acids are split off to be transported to the liver and there converted to VLDL. In type V, however, lipoprotein lipase activity is present and fatty acids can be split off; as there is a low FIAT function, such fatty acids are likely to spill over into the blood and be transported to the liver for conversion to VLDL which may be expected to appear in the blood in increased amount.

In summary, the finding of a patient with massive hypertriglyceridaemia and normal postheparin lipoprotein lipase activity who responded to both nicotinic acid and nicotinamide with normalization of plasma triglycerides led us to look for other mechanisms of hypertriglyceridaemia than low lipoprotein lipase activity. The patient was found to have a low FIAT (fatty acid incorporation into $\stackrel{0}{-}$ adipose tissue) which was corrected by treatment $\overrightarrow{\vec{F}}$ with nicotinic acid. In further studies a low FIAT was demonstrated in several types of hypertriglyceridaemia. Since removal of plasma triglycerides $\overline{\bar{\omega}}$ requires the following processes: (1) hydrolysis to $\overparen{\mathbb{D}}$ fatty acids by lipoprotein lipase and (2) uptake and esterification of the fatty acids (FIAT), we suggest $\infty$ that hypertriglyceridaemia may, among other things, $\vec{O}$ be caused by a low lipoprotein lipase activity and/or $\overrightarrow{\mid}$ a low FIAT function.

References

Carison, L. A., Eriksson, I., and Walldius, G. (1973). A case of is massive hypertriglyceridaemia and impaired fatty acid in- $\dot{\sigma}$ corporation into adipose tissue glycerides, both corrected by nicotinic acid. Acta med. scand., in press.

Carlson, L. A., Fröberg, S., and Orö, L. (1972). A case of massive $\stackrel{\vec{D}}{\Delta}$ hypertriglyceridemia corrected by nicotinic acid or nico- 0 tinamide therapy. Atherosclerosis, 16, 359-368.

Boberg, J. (1972). Heparin-released blood plasma lipoprotein lipase activity in patients with hyperlipoproteinemia. Acta med. C scand., 191, 97-102.

Boberg, J., and Carlson, L. A. (1964). Determination of heparininduced lipoprotein lipase activity in human plasma. Clin. chim. Acta, 10, 420-427.

Gustafson, A., Alaupovic, P., and Furman, R. H. (1965). Studies of the composition and structure of serum lipoproteins: isolation, purification and characterization of very low density lipo- $\omega$ proteins of human serum. Biochemistry, 4, 596-605.

World Health Organization Memorandum (1970). The classification of hyperlipidaemias and hyperlipoproteinaemias. By J. L. Beaumont et al. Bull. Wld Hith Org., 43, 891-915. 